\title{
Reorganization of Cytoskeletal and Junctional Proteins During Cochlear Hair Cell Degeneration
}

\author{
Yehoash Raphael and Richard A. Altschuler \\ Kresge Hearing Research Institute, University of Michigan \\ Medical School, Ann Arbor
}

\begin{abstract}
Experiments were carried out to elucidate changes in cytoskeletal elements and intercellular junctions in the organ of Corti, when hair cells degenerate and phalangeal scars form. Hair cell damage was induced by exposing guinea pigs to high intensity noise. The spatial and temporal changes in the organization of microfilaments, intermediate filaments, and tight junction-specific proteins were investigated using scanning and transmission electron microscopy and histochemistry. The results show that microfilaments, cytokeratins, adherens junctions, and tight junctions rearrange their distribution in damaged areas. From the temporal sequence of these changes it appears that phalangeal scars develop simultaneous with hair cell degeneration, and that the integrity of the luminal membranes in the organ of Corti is not interrupted. Each scar is formed by two supporting cells which expand and invade the sub-apical region of the dying hair cell. This region becomes cytokeratin-positive. The two supporting cells meet at the mid-line of the scar, where a new junctional complex is formed. The junctional complex consists of tight junction and adherens-type junction, but desmosomes are absent.
\end{abstract}

Key words: guinea pig, organ of Corti, cytokeratins, actin, cingulin, phalangeal scar

\section{INTRODUCTION}

Epithelial sheets establish a barrier between compartments of different ionic compositions, and regulate the composition of fluids in these compartments through selective vectorial transport of various elements [Simons and Fuller, 1985; Gumbiner, 1987]. The sensory epithelium of the inner ear, the organ of Corti, is composed of hair cells and supporting cells (SCs) which are organized in an elaborate mosaic (Fig. 1A,B). One of the roles of this epithelial mosaic is to form the barrier between two different fluid spaces, containing endolymph and perilymph. The barrier, known as the reticular lamina (Fig. (B), is formed by the apical membranes of all cells in the mosaic of the organ of Corti, and the intercellular tight junctions (TJ) between these cells [Gulley and Reese, 1976; Smith, 1978]. The luminal fluid in the organ of Corti is potassium-rich endolymph. If it were to leak into the extracellular fluid bathing the basolateral membranes of the epithelium, normal synaptic function would be interrupted and the hair cells gradually poisoned [Duvall and Rhodes, 1967]. Maintenance of the ionic barrier is therefore a sine qua non for the function and survival of the organ of Corti. Humans and experimental mammals can maintain a certain degree of hearing ability even after partial hair cell loss. This suggests that the organ of Corti can protect itself against fluid leakage, by maintaining the functional integrity of the ionic barrier during degeneration of damaged hair cells.

The mechanism which regulates repair during hair cell degeneration is probably designed to keep the ionic barrier intact. This mechanism relies on phalangeal scar formation in the reticular lamina, involving the replacement of hair cells by SCs [Bohne, 1976; Engström et al., 1966; Hawkins, 1973]. The molecular basis of the se-

Received August 22, 1990; accepted November 1, 1990.

Address reprint requests to Yehoash Raphael, Kresge Hearing Research Institute, University of Michigan, 1301 E. Ann Street, Ann Arbor, MI 48109-0506. 


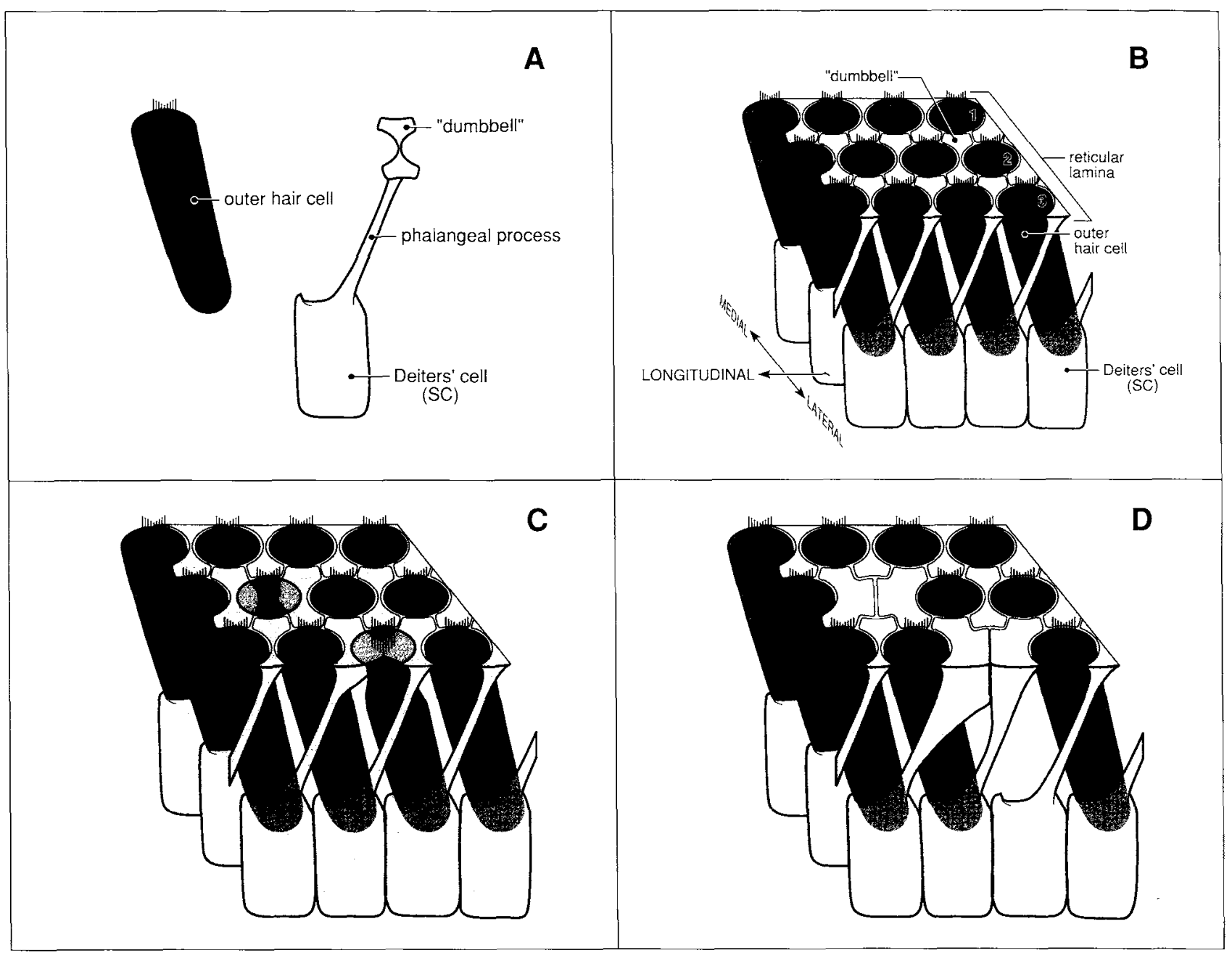

Fig. 1. Drawing of a segment of the normal organ of Corti in the OHC region. A: The tissue is composed of two cell types, OHCs and SCs (Deiters or pillar cells). The later have three distinctive domains: cell body, phalangeal process, and an apical expansion which is dumbbellshaped. B: The two cell types create a complex but orderly mosaic. The apical membranes of OHCs and SCs compose the reticular lamina. There are three rows of $\mathrm{OHCs}(1,2,3)$, running longitudinally along the cochlear spiral. Note that SCs extend their narrow processes to the reticular lamina diagonally. Thus, each $\mathrm{OHC}$ is supported by five SCs, one which envelops the basal end of the $\mathrm{OHC}$ and four

quence of events in scar formation is largely unknown. We have observed changes in the organization of actin and cytokeratins in the organ of Corti after drug induced hair cell damage [Raphael and Altschuler, 1990]. The present study was designed to investigate the temporal sequence of changes in the distribution of cytoskeletal proteins and intercellular junctions during scar formation. To induce degeneration of some outer hair cells (OHCs), guinea pigs were exposed to high intensity acoustical noise. The spatial and temporal changes in the organization of microfilaments, intermediate filaments others whose narrow process contacts the $\mathrm{OHC}$ in its apical end. C: Two stages of scar formation at the reticular lamina. An OHC in the second (middle) row is "squeezed" by two SCs which invade its sub-apical space. A more advanced stage of scar formation is depicted in a third row (hateral) $\mathrm{OHC}$, where the two scar-forming cells are attached at the mid-line. D: The two scars described in $\mathrm{C}$ are complete when the OHC is degenerated. (Modified from William E. Bronwell [1990]: Outer hair cell electromotility and otoacoustic emissions. Ear and Hearing 11:86.)

(IFs), and TJ-specific proteins were investigated after the noise exposure.

Our results show a rearrangement in the distribution of microfilaments, IFs, adherens junctions (AJs), and TJs in damaged areas of the reticular lamina. From the temporal sequence of these changes we infer that phalangeal scars develop simultaneous with $\mathrm{OHC}$ degeneration. Each scar is formed by two SCs which constrict the sub-apical region of the dying OHCs. The two SCs meet and attach to each other, forming a new junctional complex at the attachment site. The new junction is com- 
posed of $\mathrm{TJ}$ and $\mathrm{AJ}$, which are continuous with the surrounding junctions in the reticular lamina.

\section{MATERIALS AND METHODS}

Twenty young pigmented guinea pigs $(225-350 \mathrm{~g})$ were used. To induce $\mathrm{OHC}$ lesions, 16 animals were overexposed to an octave band noise with center frequency of $4.0 \mathrm{kHz}$, presented at $115 \mathrm{~dB}$ sound pressure level for 14 hours. A group of four noise-exposed animals was sacrificed upon completion of noise exposure. The other 12 noise-exposed animals were divided in three groups (four animals in each group). Animals in these three groups were sacrificed three, six or nine hours after the noise exposure. The remaining four animals served as an untreated control group. Animals were anesthetized, the temporal bones were quickly removed and immersed in the fixing solution. The osseous otic capsule was partly removed in all four cochlear turns, to allow fast perfusion of the fixative into the entire cochlear epithelium.

One animal from each group was prepared for electron microscopy analysis, one cochlea for scanning (SEM) and the other for transmission (TEM) electron microscopy. These cochleae were fixed in $2 \%$ paraformaldehyde and $2.5 \%$ glutaraldehyde in $0.15 \mathrm{M}$ cacodylate buffer, $\mathrm{pH} 7.35$, for 2 hours, and then postfixed in $1 \%$ osmium tetroxide for 1 hour. After dehydration the remaining parts of the otic capsule were removed. SEM samples were critical point dried in $\mathrm{CO}_{2}$, gold sputter coated and examined in an AMRAY 1000B scanning electron microscope. TEM samples were embedded in Epon 812, sectioned with a diamond knife on a ReichertJung Ultracut E ultramicrotome, stained with uranyl acetate and lead citrate, and analyzed in a Jeol JEM 1200 EX electron microscope. Semi-thin light microscope (LM) sections were stained with toluidine blue and photographed with a Leitz Dialux microscope.

Two animals from each group were prepared for fluorescence immunohistochemistry (two cochleae labeled for cingulin and two for cytokeratin, all four double-labeled with phalloidin for actin). Animals were fixed in $2 \%$ paraformaldehyde in $0.1 \mathrm{M}$ phosphate buffer,

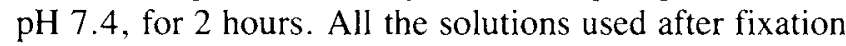
were made in PBS. After removing the surrounding otic capsule, the modiolar axis along with the organ of Corti were separated from the temporal bone. At this stage, the spiral ligament, stria vascularis and tectorial membrane were removed with fine tweezers. The cochleae were immersed in $0.1 \%$ Triton $\mathrm{X}-100$ for 5 minutes, followed by 30 minutes incubation in $4 \%$ normal goat serum to block non-specific antibody binding. Whole cochleae were incubated in primary antibody for 90 minutes at room temperature, rinsed in PBS and incubated for 30 minutes in a fluorochrome-conjugated second antibody. After a final rinse, the epithelium of the organ of Corti was separated from the bony modiolus, dissected in halfcoils and mounted in Gelvatol 20/20 (Monsanto, St. Louis).

Cochleae from one animal in each group were immunostained for cytokeratin $(\mathrm{CK})$ proteins, with the ABC-peroxidase method [Hsu et al., 1981], using Vectastain $\mathrm{ABC}$ Kit from Vector Laboratories (Burlingame, CA). Fixation and incubations with blocking solution and primary antibodies were performed as described above for immunofluorescence, except that the blocking buffer consisted of 5\% normal horse serum. These preparations were initially mounted in glycerol for whole-mounts and later embedded in Epon and sectioned with glass knives for LM analysis.

Extensive control experiments were performed to verify antibody specificity for both fluorescence and peroxidase methods, using guinea pig cochleae as well as cultured guinea pig cells. In control experiments, primary antibodies were replaced by PBS or nonimmune sera.

Monoclonal anti-CK pan antibody, which binds to an epitope common to all types of CKs, was purchased from Boehringer Mannheim Biochemicals (Indianapolis, IN). Monoclonal anti-cingulin [Citi et al., 1988] was kindly supplied by Dr. Sandra Citi (Cornell University Medical School, New-York). FITC- and rhodaminc-conjugated goat-anti-rabbit and goat anti-mouse second antibodies were purchased from Boehringer Mannheim. To stain actin filaments, rhodamine- or FITC-conjugated phalloidin (Molecular Probes, Junction City, Oregon) was used at 1:30 dilution in the second antibody solution. The tissue was examined and photographed using a Leitz Orthoplan microscope equipped for epifluorescence. Photographs were taken on Kodak Tmax 400 film, exposed at 1600 ASA.

\section{RESULTS}

\section{Temporal Determination of Scar-Formation}

The cells which compose the reticular lamina and the epithelial mosaic of the organ of Corti are depicted in a drawing (Fig. 1A,B). To define the temporal sequence of changes in the organ of Corti after noise exposure, we compared untreated controls to noise exposed animals. The normal pattern of actin-specific staining in the apical domains of cells or the organ of Corti is demonstrated in a whole-mount preparation labeled with phalloidin (Fig. 2). Viewing the reticular lamina from above, actin-specific labeling is seen in the stereocilia of hair cells, in the cuticular plate (terminal web) of these cells and in the AJ-complexes. AJs contain a circumferential bundle of actin which surrounds each cell in the reticular lamina (for orientation and definition of planes, see Fig. 1). 


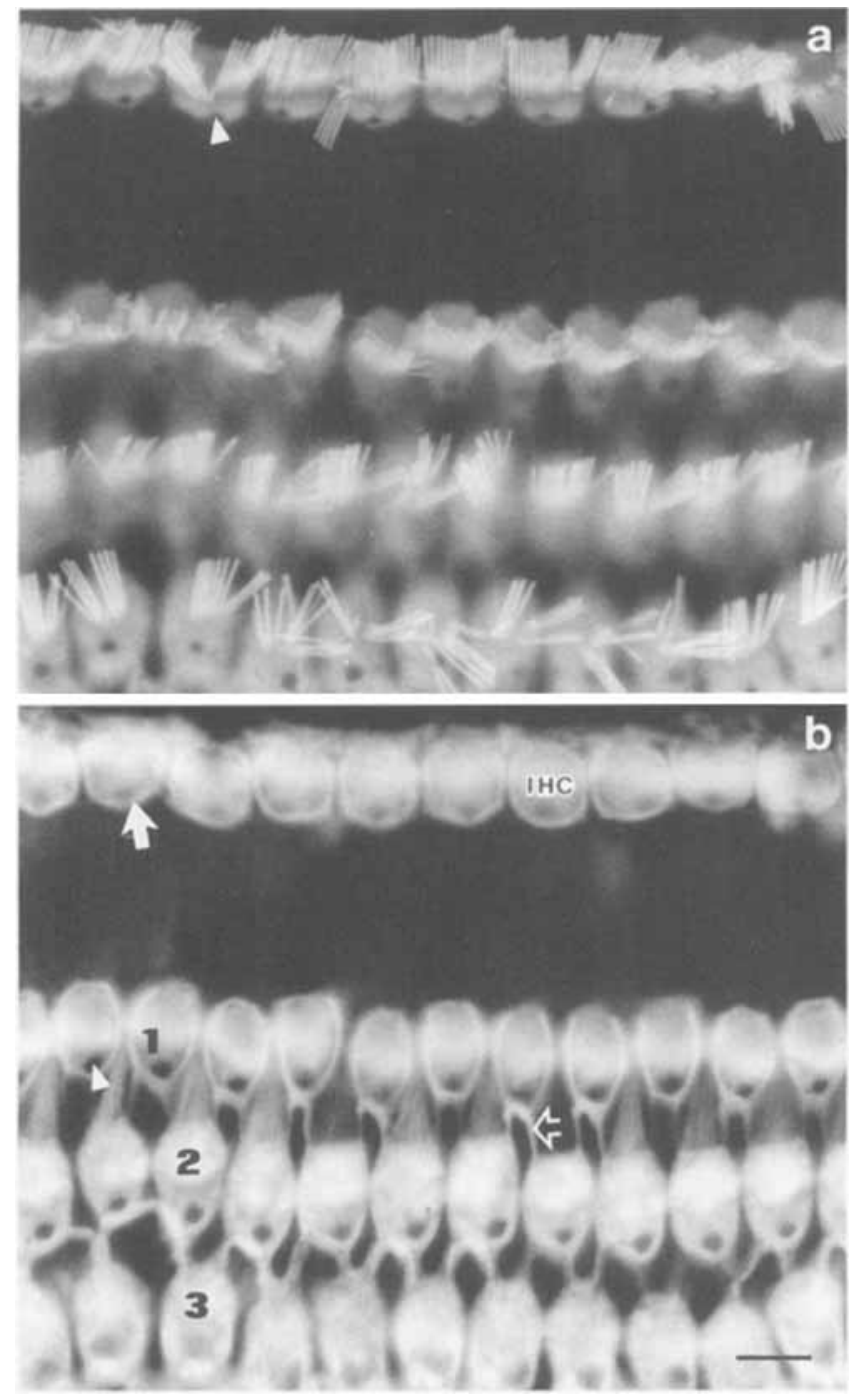

Fig. 2. Surface view of a phalloidin-labeled whole-mount of control organ of Corti, photographed at two different focal planes. a: Focal plane showing mainly stereocilia of inner hair cells and three rows of OHCs. Arrowhead: Basal body area of an inner hair cell. b: The focal plane is more basal than a, showing the reticular lamina. IHC =inner hair cell; solid arrow = actin in AJ around an inner hair cell; open arrow $=\mathrm{SC}-\mathrm{SC} \mathrm{AJ}$; arrowhead $=$ actin free zone in cuticular plate (basal body area) of first row OHC. 1, 2, 3 = three rows of OHCs. Bar, $10 \mu \mathrm{m}$.

There is an actin-free area in the apical region of hair cells, where a basal body is usually present [Engström et al., 1962; Flock et al., 1962].

CK-specific staining in the reticular lamina of control animals is restricted to the apical domains of SCs [Raphael et al., 1987]. Surface view of the reticular lamina labeled for cytokeratins using the $\mathrm{ABC}$-peroxidase method revealed positive immunoreactivity in the apical domains of SCs, shaped like dumbbell (Fig. 4a, compare to $1 \mathrm{~B})$. CKs are also apparent at a deeper focal plane in the phalangeal process (Fig. $4 \mathrm{~b}$ ). Cross sections of the organ of Corti confirm that CK-specific label appears in the apical part of Deiters cells but not OHCs (Fig. 6a, and higher magnification, $b$ ).

Cingulin, a protein component associated with tight junctions, has been found in junctional complexes of various epithelia [Citi et al., 1988, 1989]. To localize TJs in the reticular lamina, fluorescent labeling of whole-mounts with anti-cingulin antibodies was performed. Viewing control organ of Corti preparations from above, anti-cingulin staining appears as a distinct, uninterrupted line which forms a mosaic of circles and dumbbells on the apical surface of OHCs and SCs, respectively (Fig. 7a).

The pattern of staining observed in the majority of degenerating cells was compared between animals allowed to survive $0,3,6$ and 9 hours after noise exposure, to determine the sequence of changes at the reticular lamina.

Stage 1. The first stage of changes in the reticular lamina of noise-exposed animals is typically observed within 3 hours after exposure. A substantial reduction in actin-label is found in the stereocilia and the cuticular plate (Fig. 3a). In contrast, actin staining associated with $\mathrm{AJ}$ appears normal at this stage. No change is observed in the pattern of CK-specific label or in the distribution of cingulin in the reticular lamina.

Stage 2. The second stage is typically found in animals which survived 6 hours after noise exposure and involves changes in staining for actin, $\mathrm{CKs}$, and cingulin. Sites of damaged OHCs in the reticular lamina contain two actin-positive bands oriented in the plane of the reticular lamina (Figs. 1C, 3b). The area of damaged cells in the group of animals which survived 6 hours, typically contains bands whose central regions bend toward each other, creating an hourglass shape (Fig. 3c). These bands gradually come nearer to each other, until they appear parallel, oriented in the plane of the reticular lamina in the medial-lateral axis (Figs. 1C, 3c). The area thought to contain the basal body of the $\mathrm{OHC}$ remains actin-free.

The distribution of CKs in the reticular lamina is also altered at the second stage. The apical region of damaged OHCs becomes CK-positive, apparently due to expansion of the $\mathrm{SC}$ into the space normally occupied by the OHC (Figs. 1C, 4c,d). Phase-contrast inspection of CK labeled scar areas revealed that several damaged OHCs still have a bundle of stereocilia extending from their apical process (results not shown). To correlate the temporal change in CK distribution with that of actin, whole-mounts of the organ of Corti were co-labeled with two fluorescent tags, one for CKs and one for actin (Fig. 5 , fluorescent signal is bright and background is dark). OHCs which have normal actin distribution (Fig. 5b) are 

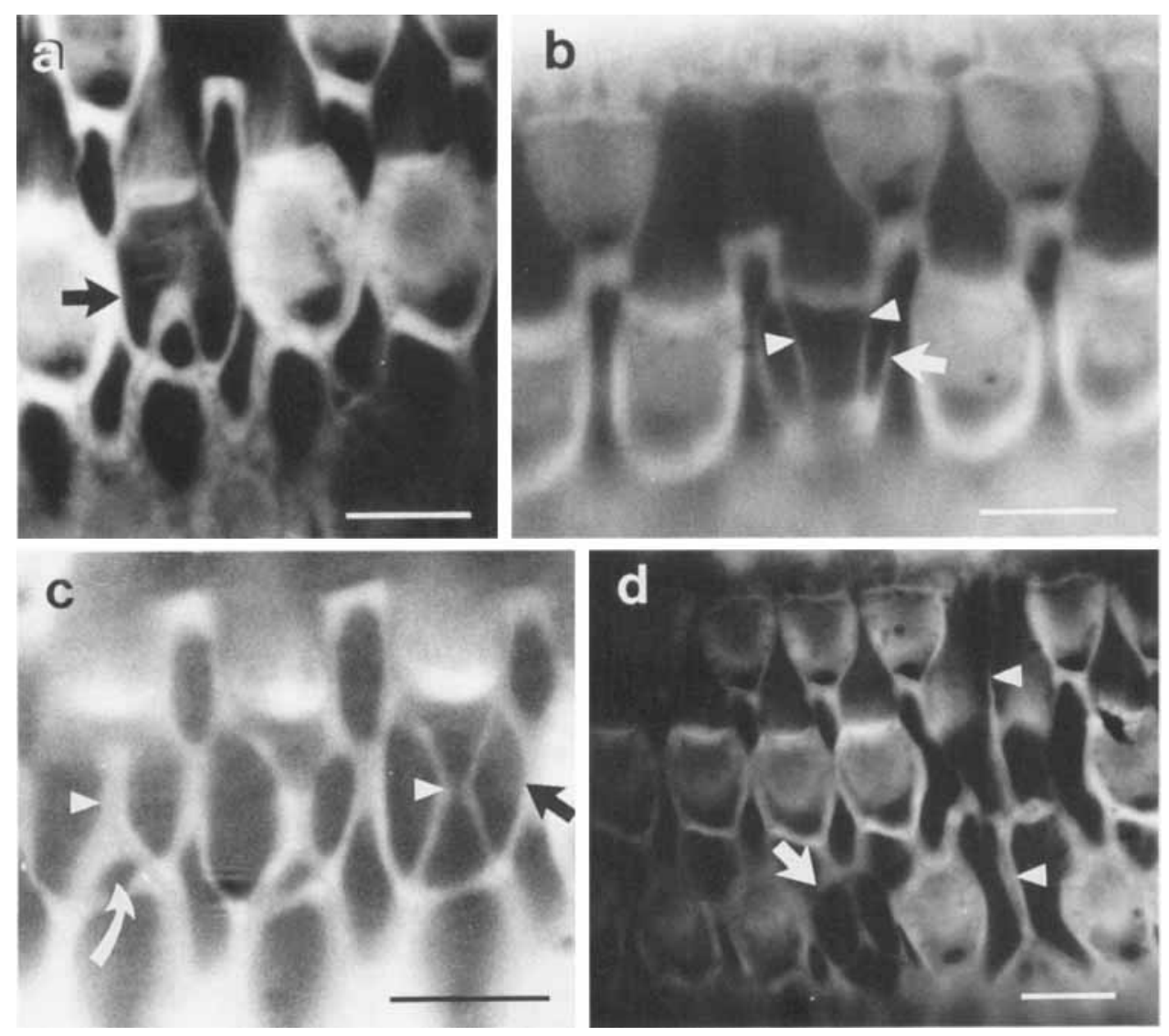

Fig. 3. Surface view of phalloidin-labeled whole-mounts at four stages of OHC degeneration. a: Three hours after the noise exposure, a reduced staining for actin in the apical domain marks damaged OHCs. Arrow: AJ between a damaged cell (on the right) and a relatively normal looking cell (on the left). b: Six hours after the noise, two actin-labeled bands (arrowheads) are seen in the region of damaged OHCs. Actin in the original OHC AJ is still visible (arrow). c: Three damaged OHCs at different stages of scar formation. The two actin-bands approach each other forming an hourglass shape (arrow-

surrounded by dumbbell-shaped SCs (Fig. 5a, compare to Fig. 1B, 4a). In contrast, where the distribution of actin marks a damaged OHC (Fig. 5b), CK-positive area of adjacent SCs appears to have expanded into the space of the damaged OHC (Fig. 5a). Some actin staining is still present in the AJ of the OHC (Fig. 5b). Taken together, the observations based on $\mathrm{CK}$ and actin labeling, combined with phase contrast analysis, indicate that CKpositive areas are located beneath the apical membrane of degenerating OHCs.

To better understand the relationship of $\mathrm{CK}$ organization in the reticular lamina to the three-dimensional structure of the organ of Corti, Epon-embedded, CKlabeled whole-mounts were sectioned at the mid-modiolar plane (Fig. 6). After noise exposure, scars which have replaced damaged $\mathrm{OHCs}$ at the reticular lamina are CK-positive (Fig. 6c). An area of CK-negative cyto- head in cell on the right). The two actin-bands gradually attach to each other (cell in center and left arrowhead). The cuticle-free area of $\mathrm{OHCs}$ is devoid of actin stain (white arrow). Actin-specific label is present in original OHC AJ (black arrow). d: Typical actin distribution found in most scars 9 hours after the noise exposure. New AJ (arrowheads) connect two scar-forming SCs. In another scar which is in earlier stage of formation (arrow) the original $\mathrm{AJ}$-associated actin label is still visible. Bars, $10 \mu \mathrm{m}$.

plasm, which could belong to a degenerating $\mathrm{OHC}$, is detected adjacent to the lumen. In some scars, CK-positive cytoplasm occupy the entire apical region of the scar. In addition, the pattern of CK-label shows that the stalk of the $\mathrm{SC}$ is enlarged in diameter (Fig. 6d, compare to Fig. $4 b$ ).

During the second stage of scar formation, a new cingulin-positive line appears (Fig. 7b). Co-labeling for actin verified that the position of the new cingulin line corresponds to the actin in the new SC-SC junction (Fig. 7c). In the original OHC-SC junctional complex, TJ labeling is absent when AJ-associated actin-specific staining is still present. The area normally occupied by the basal body of the OHC is devoid of cingulin and actin staining (Fig. $7 \mathrm{~d}, \mathrm{e}$ ).

TEM analysis of cross-sections through scarred areas revealed that the apical surface of the two scar- 

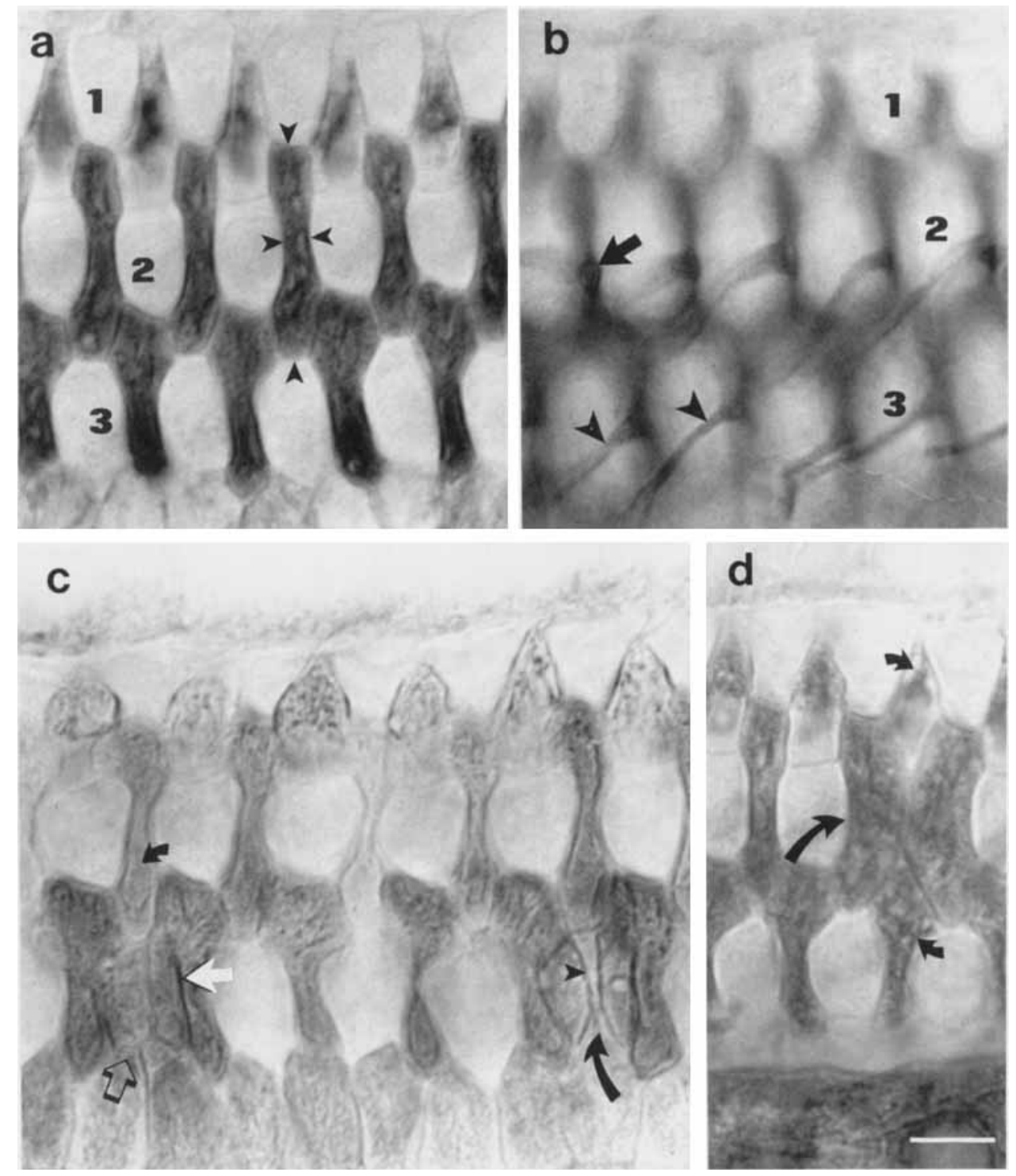

Fig. 4. CK-specific labeling in normal $(\mathbf{a}, \mathbf{b})$, and noise exposed $(\mathbf{c}, \mathbf{d})$ organ of Corti, as determined by surface view of ABC-peroxidase processed whole-mounts. a: At the reticular lamina level, OHCs of all three rows (1-3) are devoid of CK-specific label, while SCs are heavily labeled, forming a dumbbell shape (borders of one SC are marked by four arrowheads). b: At a focal plane beneath the reticular lamina, CK-positive label appears in the phalangeal process which narrows (arrow) and descends in diagonal-basal direction (arrowheads) toward the cell body (compare to Fig. 1). c: Two scars in third row OHCs 6 hours after noise exposure. SCs from same row in the reticular lamina expand and invade space of OHCs (large curved arrow). A dense dark line (arrowhead) is present in border of CKpositive area and in original $\mathrm{AJ}$ of the $\mathrm{OHC}$. In cell on left (empty arrow) $\mathrm{CK}$ label covers the entire scar, but a dense line is still visible in original AJ (white arrow). Note that shape and size of SC medial to the scar (small curved arrow) is similar to other SCs in the same row. d: Nine hours after the noise exposure most scars are entirely stained for CKs (big arrow) and the dense line in the original $\mathrm{AJ}$ is no longer observed. SCs in lateral and medial position relative to the scar (small arrows) remain unaltered. Bar, $10 \mu \mathrm{m}$.

The phalangeal scar. Nine hours after noise exposure, most of the degenerating OHCs have been replaced by scars. The scars are composed of the expanded apical processes of two SCs which are attached by a new junctional complex (Figs. 1D, 3d, 4d). The belt of AJ, which originally encircled the $\mathrm{OHC}$ is disassembled. The two SCs which had previously contacted the (now dead) 

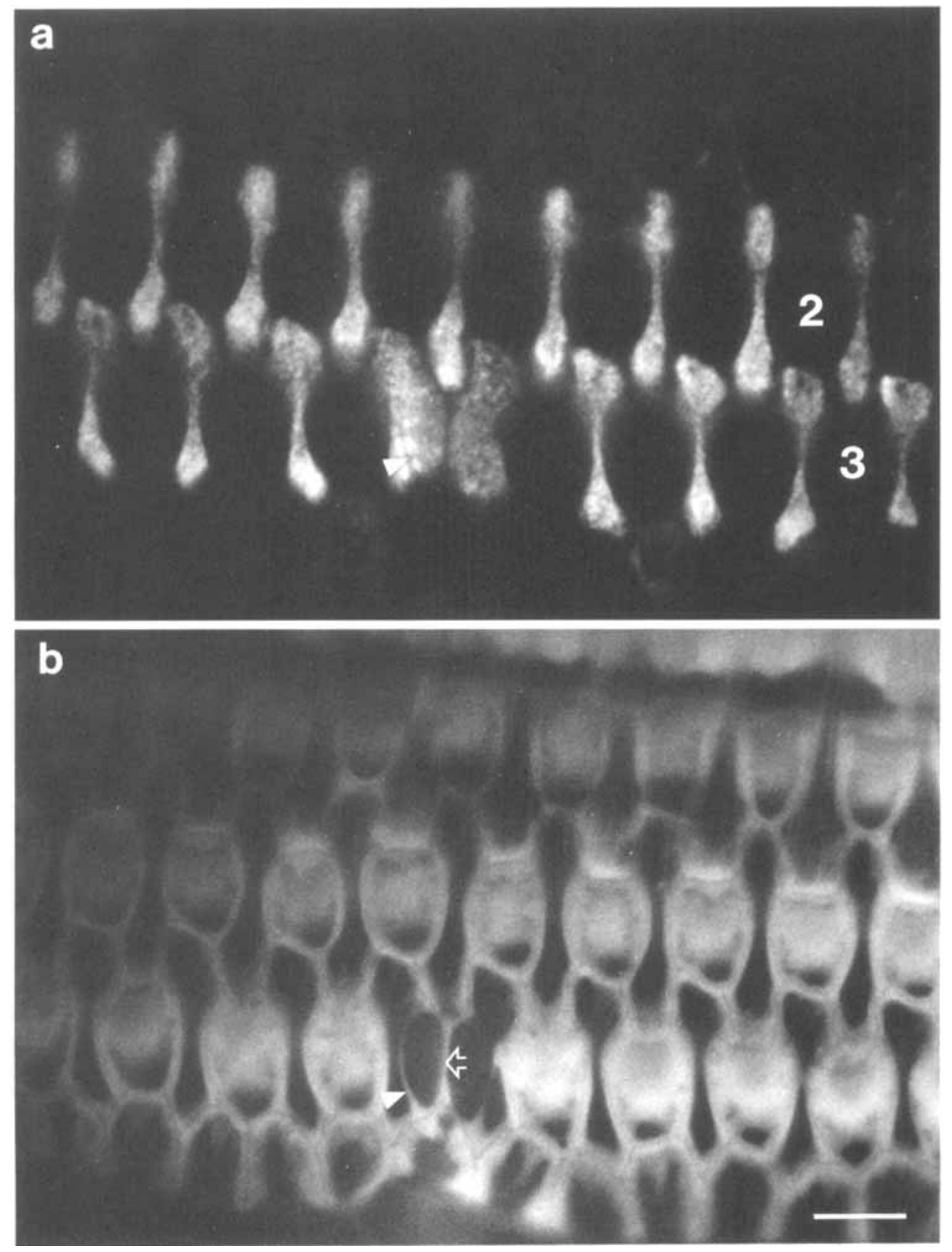

Fig. 5. Whole-mount of organ of Corti 6 hours after noise, co-labeled with two tags, one for CK (a) and the other for actin (b), photographed at the level of the reticular lamina. a: CK-specific label is restricted to SCs and not found in OHCs (2, $3=$ apical surfaces of OHCs in second and third row, respectively). CK label is found in area of one $\mathrm{OHC}$ of

OHC on its lateral and medial sides, do not change in shape or size during scar formation.

A high magnification micrograph of the apicalmost portion of the junctional complex reveals a junctional complex above the AJ (Fig. 8b). It appears like a TJ complex, although the length of the periodic mem- third row (arrowhead). b: Normal actin distribution is seen in most cells, but a forming scar is in one cell, corresponding to the area of $\mathrm{CK}$ invasion in a. A band of actin is found in the mid-line of the forming scar (open arrow) while the AJ-associated actin label is still visible in the original OHC border (arrowhead). Bar, $10 \mu \mathrm{m}$.

brane contacts was unusually extended. Desmosomal complexes are not detected in the new junctional complex. SEM analysis of the reticular lamina show scattered scars in different regions along the cochlear duct (Fig. 9). The attachment points between scar-forming SCs appear at the mid-line of each scar and protrude 

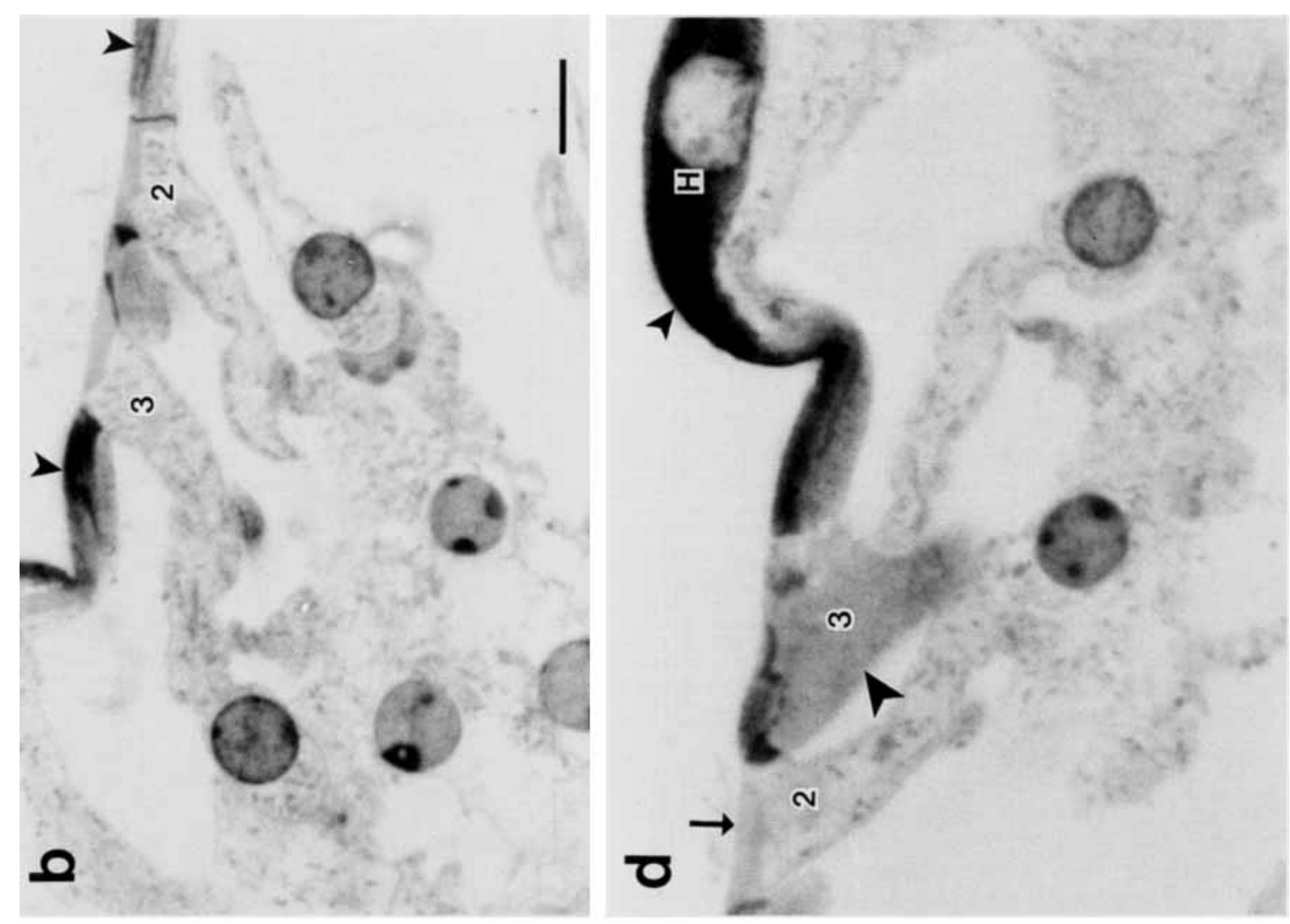

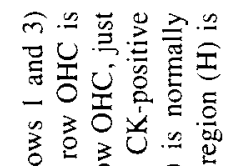

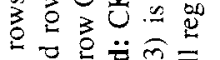

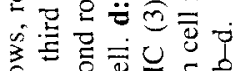

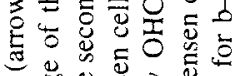

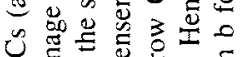

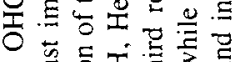

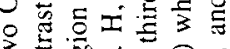

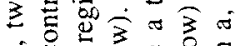

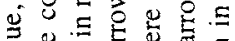

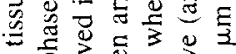

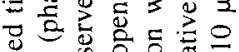

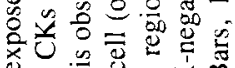

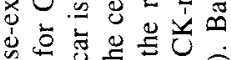

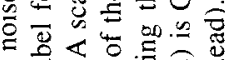

巨西

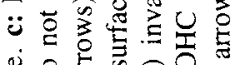

요의

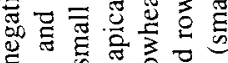

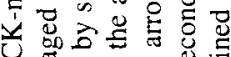

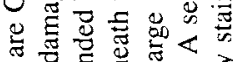

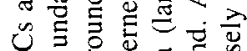

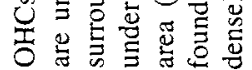

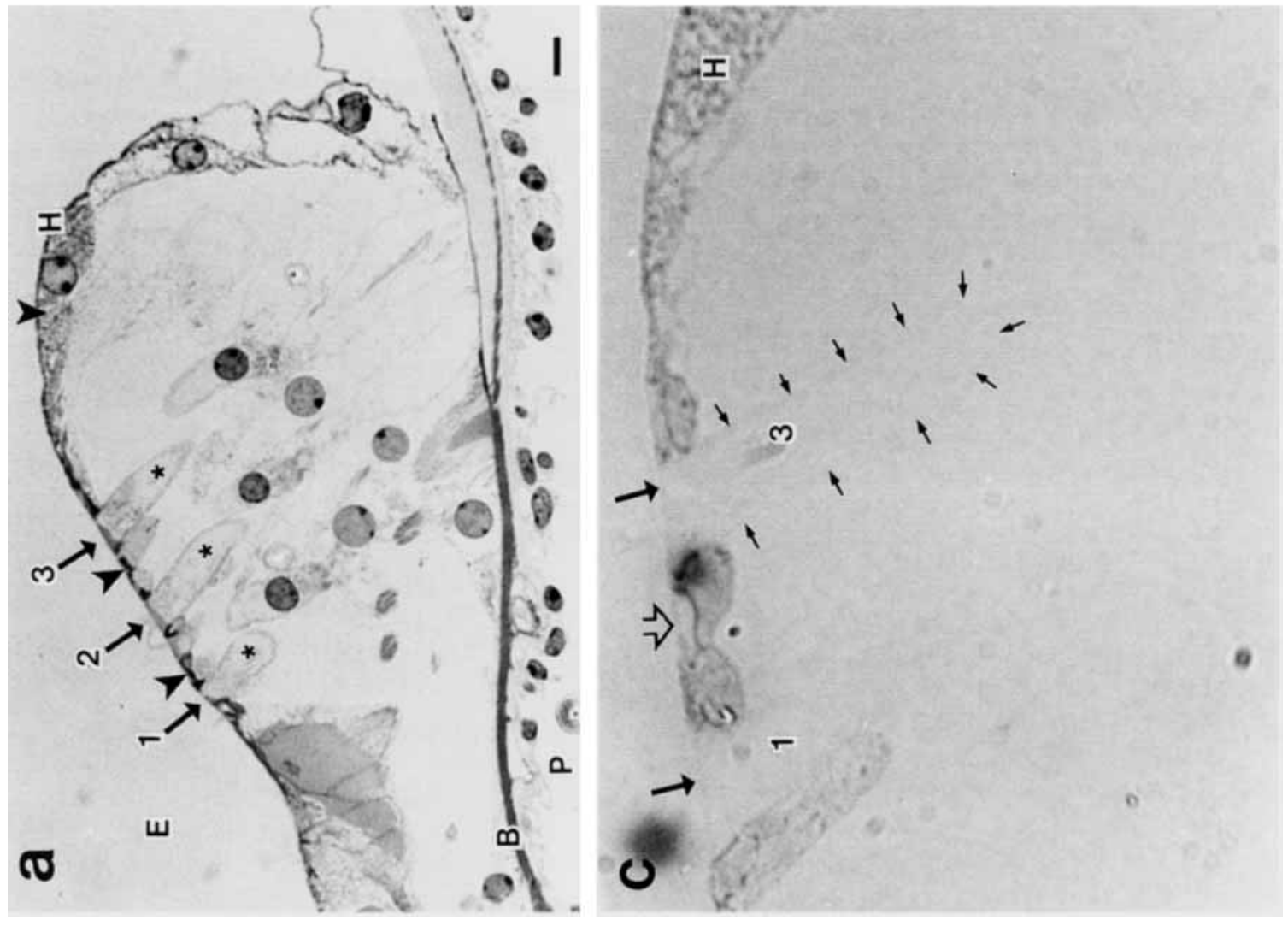

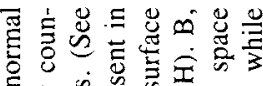

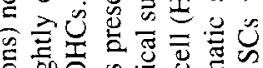

的O

要

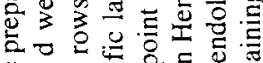

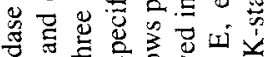

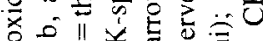

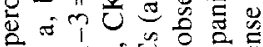

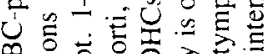

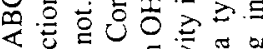

䓎

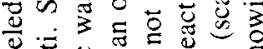

등

U

论

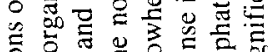

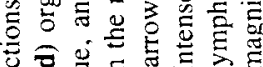

论

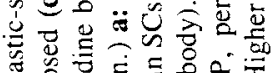

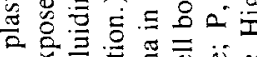

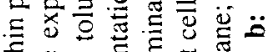

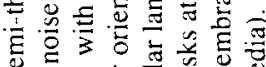

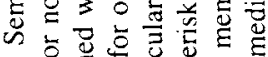

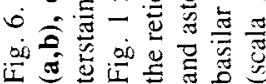



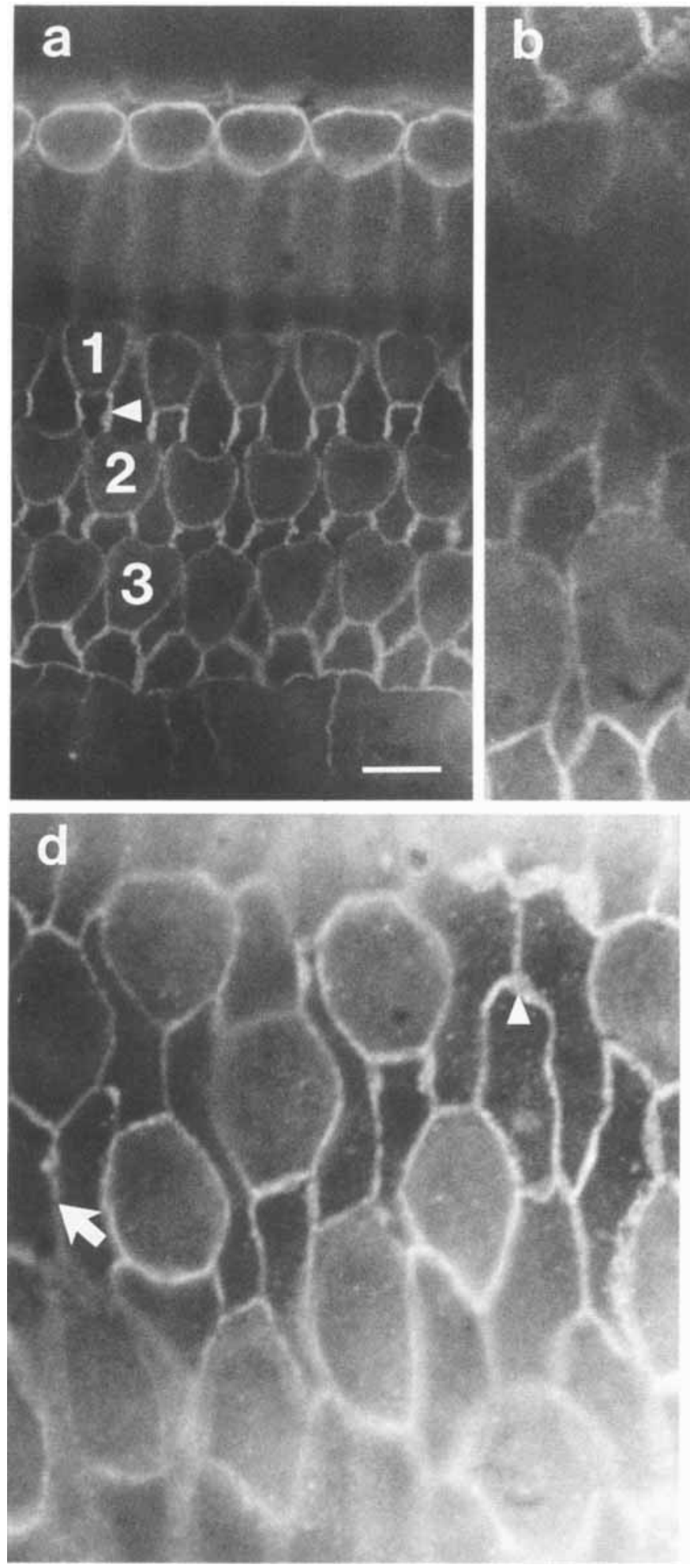

Fig. 7. Distribution of cingulin immunoreactivity in the reticular lamina in surface view of the normal organ of Corti (a) and co-labeling for cingulin and actin in noise-exposed ears (b,c and $\mathbf{d , e}$ ). a: Cingulin staining is normally found in homologous as well as heterologous junctions, interconnecting the apical junctions of all cells in the reticular lamina. The pattern of staining delineates the spherical shape of the OHCs $(\mathrm{I}-3=$ three rows of $\mathrm{OHCs})$, while the dumbbell-shaped SCs appear between them. Arrowhead: SC-SC junction. b,c: Six hours after noise exposure, reorganization of actin can be seen in a region of a degenerating $\mathrm{OHC}$ (c). A new cingulin-specific line
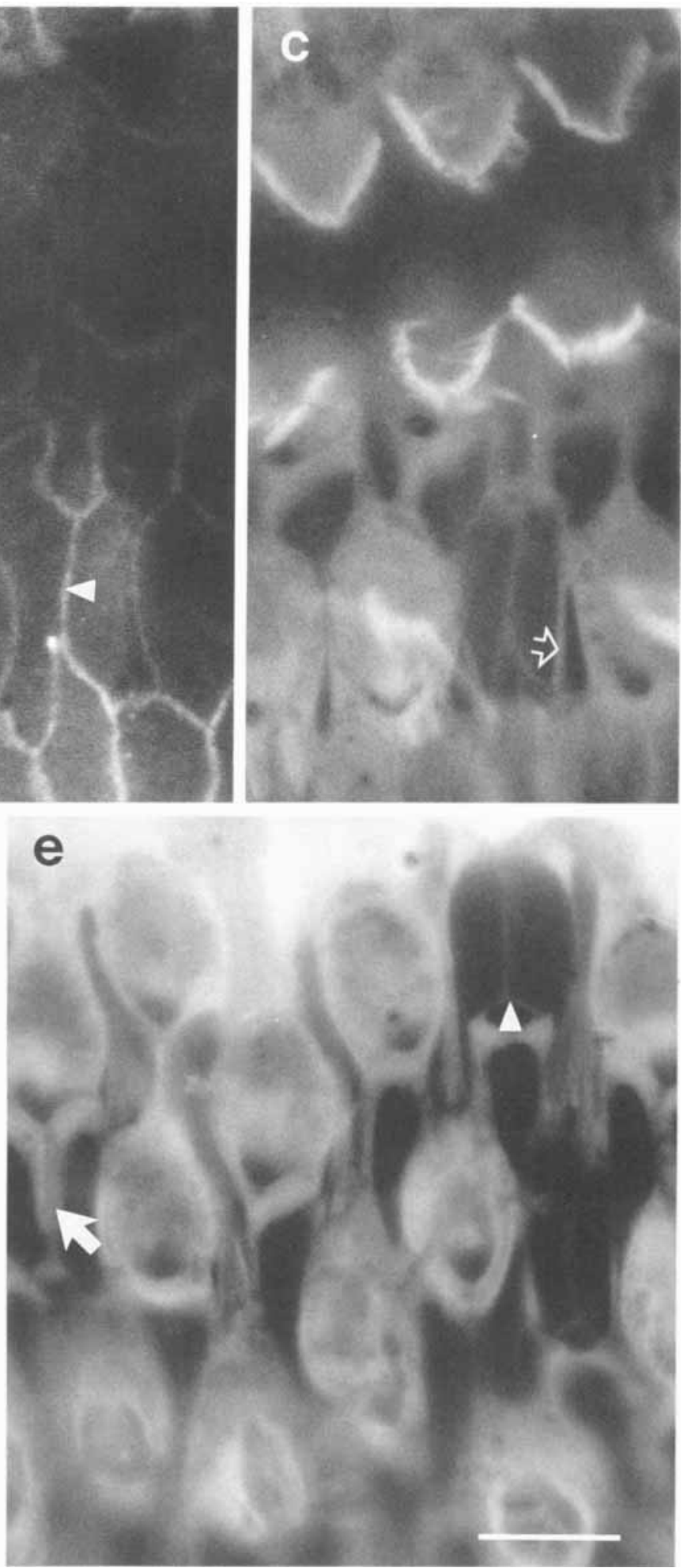

(arrowhead in $b$ ) is present in an orientation corresponding to the new adherens junction in $\mathrm{c}$. A faint actin-specific staining is present in the original $\mathrm{AJ}$ of the $\mathrm{OHC}$ (open arrow in $\mathrm{c}$ ) but cingulin is absent in this area (compare to b). d,e: Nine hours after the noise exposure a new AJ is seen in the mid-line of the scar (arrow in e), while no staining is present in the original AJ of the $\mathrm{OHC}$. TJ-specific label is oriented similar to the actin in the AJ (arrow in d). Note that both cingulin and actin form a loop around the former cuticle-free area (arrowheads in $d$ and e). Bars, $10 \mu \mathrm{m}$ in a. and in e for b-e 


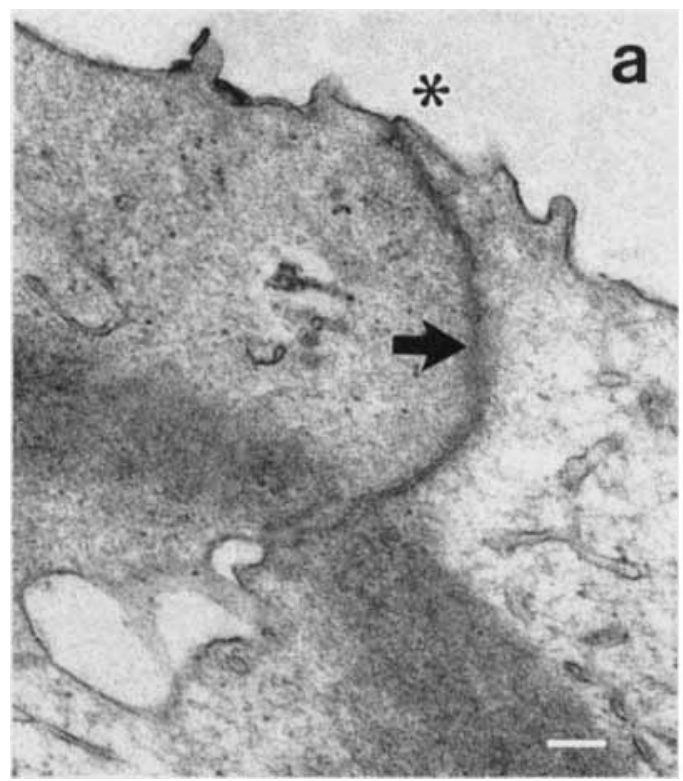

Fig. 8. TEM micrographs of scar region in the reticular lamina, sectioned in the longitudinal plane. a: Six hours after the noise insult, the contact area between two SCs appears like a junctional complex (arrow). The distribution of microfilaments in the lower part of the

above the level of the reticular lamina, similar to other junctions in the organ of Corti. In regions where two adjacent $\mathrm{OHCs}$ in a given (longitudinal) row are degenerated, each SC forms two scars. SEM analysis confirmed that of the four SCs which surround a given $\mathrm{OHC}$, only two neighbors (on the longitudinal axis) contribute to the scar. There is no significant change at the reticular lamina in the surface area or shape of the medial and neighboring SCs which reside lateral and medial to the degenerating OHC (Figs. 1D, 4d, 9).

\section{DISCUSSION}

Healing of injury in tissues is directed at preventing additional, progressive damage, as well as restoring function. In higher vertebrates, the potential to regenerate cells and to recover function is limited to very few tissue types. This repair ability is crucial for epithelial sheets which cover the body and line its cavities, to protect the body from external insults and maintain ionic environments in the various cavities. For that purpose, most epithelial tissues undergo true regeneration, based on divisions in a population of basal (stem) cells. The sensory part of the mammalian auditory epithelium, the organ of Corti, is an exception in that it is unable to regenerate lost OHCs. Instead, to repair the damage and maintain the functional continuity of the organ of Corti, non-sensory cells replace damaged OHCs in a process known as phalangeal scar formation.

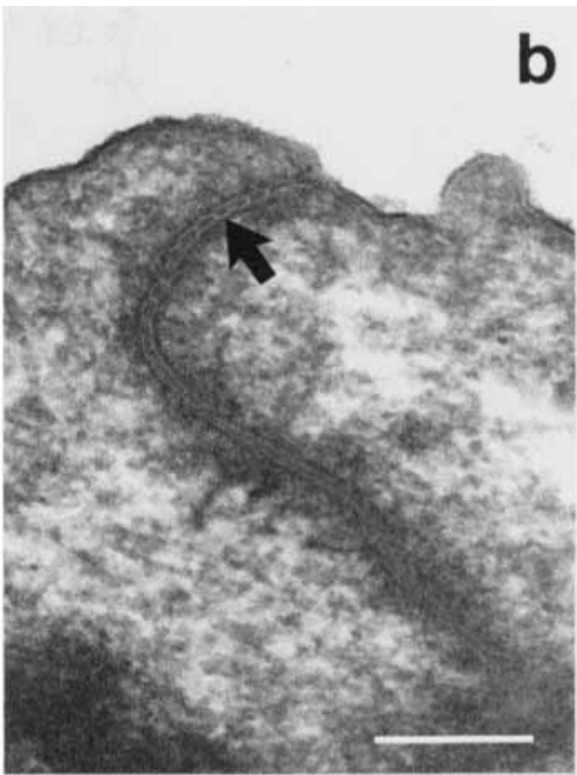

junction is relatively symmetric. Asterisk-luminal side (endolymph). b: Nine hours after the noise, a TJ (arrow) can be distinctively seen in the apical region of the junctional complex. Bars, $0.1 \mu \mathrm{m}$.

Phalangeal scars are formed by two SCs which invade the space previously occupied by the OHC. In certain circumstances, discontinuities (perforations) may temporarily occur in the reticular lamina during the process of scar formation [Bohne, 1976; Bohne and Rabbitt, 1983]. Other reports demonstrate that ototoxic or mechanical cochlear insults may cause OHC loss and phalangeal scar formation without perforations in the reticular lamina [Duvall and Rhodes, 1967; Hawkins, 1973; Forge, 1985]. Consistent with this idea, one recent study showed that the tracer-molecule lanthanum did not permeate through the reticular lamina during drug-induced OHC degeneration [McDowell et al., 1989].

Our results describe highly regulated reorganization of cytoskeletal elements in the reticular lamina during phalangeal scar formation. It appears that cochlear SCs start to form a scar before the damaged $\mathrm{OHC}$ degenerates, so that the process of scarring coincides temporally and spatially with $\mathrm{OHC}$ degeneration. This suggests that the regulation of scar formulation is coupled to OHC degeneration. When attempting to determine the source of signals which initiate the scarring process, the cytoarchitecture of the organ of Corti should be considered. The cytoarchitecture is such, that the contact between scar-forming cells and a given degenerating $\mathrm{OHC}$ is restricted to the apical junctions (see Fig. 1). This suggests that the signals for scarring are mediated via the apical contact areas.

Several reasons lead us to believe that within the 


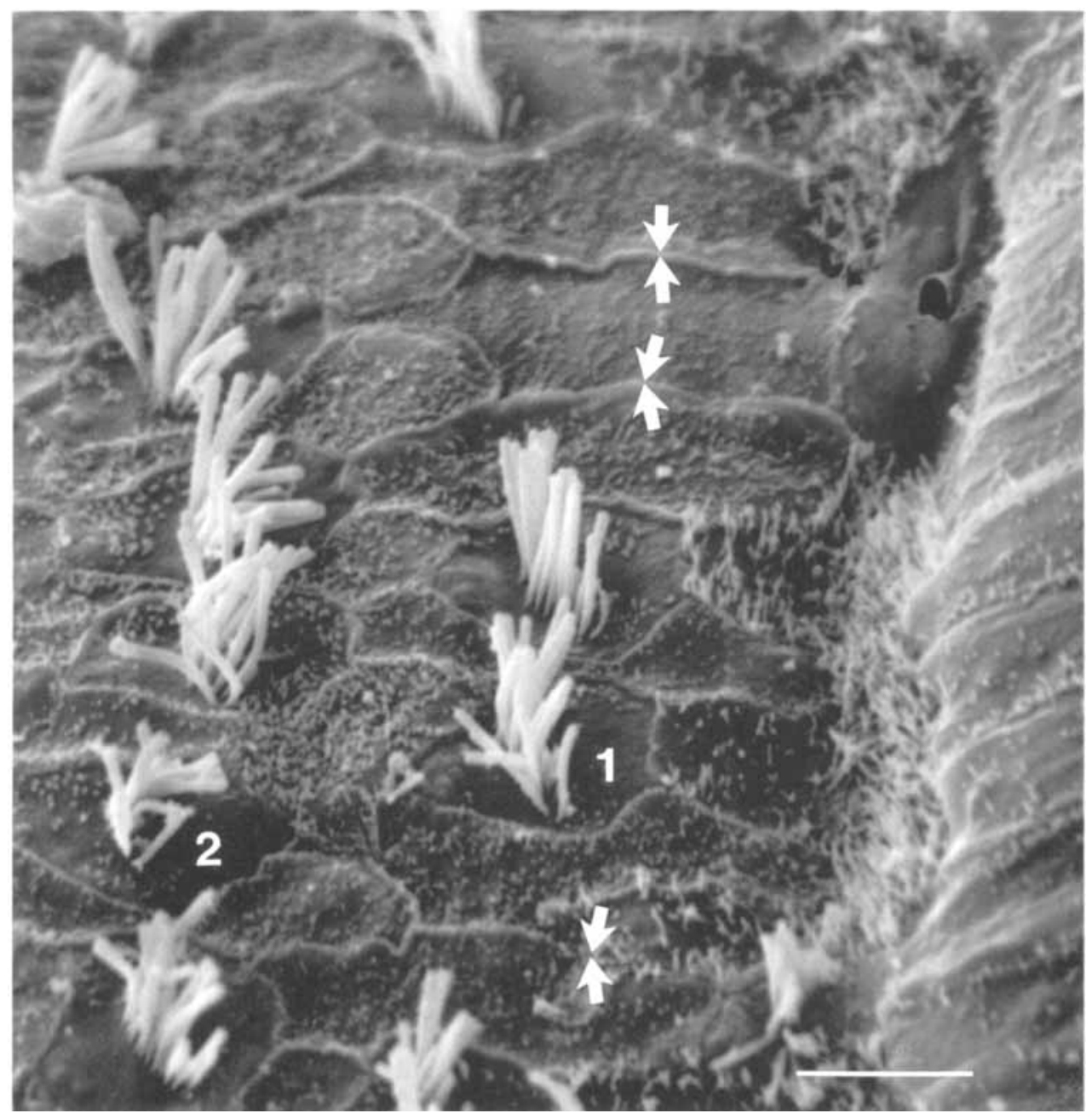

Fig. 9. SEM micrograph viewing the reticular lamina from above. Nine hours after noise exposure several scars can be discerned in the first row of OHCs (1). Each pair of arrows points at the newly formed contact area at the mid-line of the scar. Note the regular pattern of two adjacent scars. $2=$ second row OHCs. Bar, $5 \mu \mathrm{m}$.

apical contact area, AJs and the microfilament system are involved in the orientation and regulation of scar formation:

a. In the apical contact area between OHCs and SCs desmosomes are absent [Gulley and Reese, 1976], and therefore intercellular mechanical attachment and support may depend entirely on the belt of AJ.

b. AJs are considered to be involved not only in intercellular support, but also in orientation and communication [Geiger et al., 1984; Geiger et al., 1985].

c. Actin has been shown to play a role in regenerative responses in several tissues [Low et al., 1981; Gabbiani et al., 1984; Hergott et al., 1989]. d. We now demonstrate that in the organ of Corti, actin filaments rearrange in the early stages of $\mathrm{OHC}$ damage. Later, actin bands mark the edges of SCs which expand and invade the sub-apical area of the OHCs. These bands attach to each other to form an adherens junction at the new contact area.

The major changes in the organization of microfilaments after noise damage are likely to influence the mechanical balance of forces in the reticular lamina. We speculate that changes in tensile forces in the apical domains of damaged OHCs may constitute a signal which initiates scar-forming activity in neighboring SCs. The response of SCs to the microfilament changes in 
OHCs may be mediated by the AJs. Future experiments using in vitro preparation will help elucidate the role of cortical tension in regulating the process of hair cell degeneration and scar formation.

It is likely that the band of actin at the edge of the invading SCs is similar to a leading edge or, alternatively, could regulate the shape of the expanding SCs in a similar way to actin-edge-bundles in fibroblast cultures [Zand and Albrecht-Buehler, 1989]. In addition, it is known that cells may segregate junctional proteins in their membrane in advance [Geiger et al., 1985; Takeuchi, 1987]. The bands of actin in SCs may indicate that the cells prepare junctional building-blocks in advance. If true, this shows that in this case, contact with the target is not needed for determination of the future junction site.

When the two scar-forming SCs meet, the two actin-bands fuse into one line, which is continuous with actin in the AJ of adjacent cells in the reticular lamina, suggesting that an $\mathrm{AJ}$ has been formed. TEM analysis and the presence of cingulin immunoreactivity indicate that $\mathrm{TJ}$ has been established. Our results indicate that when new junctional complexes are formed, TJ appear simultaneously with AJs. It appears that the presence of a desmosome in the contact area is not required for the assembly of both TJ and AJ. Desmosomes are prominent in SC-SC junctions [Gulley and Reese, 1976]. Therefore it was surprising not to detect desmosomes in the new scar-junction. A study with longer survival times after noise exposure will be required to determine whether desmosomes appear in SC-SC junctions at a time later than 9 hours post-noise exposure.

It will be interesting to determine whether new proteins are synthesized during scar formation or an existing pool of proteins is redistributed. Low et al. [1981] suggest that changes in actin during epithelial regeneration reflect changes in the degree of polymerization rather than total amount of actin. In situ hybridization methods may prove useful to find out if and where actin synthesis takes place.

We propose the following scenario for the sequence of cytoskeletal changes and their role in noise induced injury to the organ of Corti. Noise overstimulation causes depolymerization of actin in the stereocilia, cuticular plate and AJs of OHCs. This alters the tensile forces in the reticular lamina. SCs respond by creating a bond of actin along their border with the degenerating $\mathrm{OHC}$, expanding their apical domains and gradually invading the sub-apical area of damaged OHC. SCs fuse with the degenerating OHCs and phagocytose their cytoplasm. Certain components of the degenerating OHC, perhaps the basal body, may play a role in organizing and orienting the scar. The invading SCs meet at the mid-line of the scar region and establish a new junctional complex composed of $\mathrm{TJ}$ and $\mathrm{AJ}$.
In conclusion, the results reveal a sequence of changes in the distribution of microfilaments and IFs in the reticular lamina after high-intensity noise exposure. Actin filaments disappear from stereocilia and the cuticular plate of OHCs. CK-rich SCs invade the sub-apical space of damaged OHCs. A new junctional complex is formed where the invading SCs meet, beneath the apical membrane of the $\mathrm{OHC}$, so that the continuity of the reticular lamina is not interrupted. The pattern of cytoskeletal rearrangement during phalangeal scar formation suggests that $\mathrm{OHC}$ degeneration coincides temporally with scar formation. The results suggest that SCs can replace damaged OHCs without interrupting the integrity of the reticular lamina.

\section{ACKNOWLEDGMENTS}

We wish to thank Chris Sheridan for skillful TEM processing, Linda Gafney for excellent dark-room work, and Dr. Cynthia Prosen for assistance in noise exposure. We acknowledge the kind gift of anti-cingulin antibodies from Dr. Sandra Citi, and are grateful for the helpful comments made by Drs. Stephen Ernest, Benjamin Geiger, and Martin Zand, who critically read the manuscript. This work was supported by NIH grant RO DC 00383.

\section{REFERENCES}

Bohne, B.A. (1976): Healing of the Noise Damaged Inner Ear. In Hirsh, S.K., Eldredge, D.H. Hirsh, I.J. and Silverman, S.R. (eds.): "Hearing and Davis: Essays Honoring Hallowel Davis". Saint Louis, Washington University Press, pp. 85-96.

Bohne, B.A., and Rabbit, K.D. (1983): Holes in the reticular lamina after noise exposure: Implication for continuing damage in the organ of Corti. Hearing Res. 11:41-53.

Citi, S., Sabanay, H., Jakes R., Geiger, B., and Kendrick-Jones, J. (1988): Cingulin, a new peripheral component of tight junctions. Nature 333:272-276.

Citi, S., Sabanay, H., Kendrick-Jones, J., and Geiger, B. (1989): Cingulin: characterization and localization. J. Cell Sci. 93: $107-122$.

Duvall, A.J., III, and Rhodes, V.T. (1967): Ultrastructure of the organ of Corti following intermixing of cochlear fluids. Ann. Otol. Rhinol. Laryngol. 76:688-708.

Engström, H., Ades, H.W., and Hawkins, J.E. (1962): Structure and functions of the sensory hair cells of the inner ear. J. Acoust. Soc. Am. 34:1356-1363.

Engström, H., Ades. H.W., and Andersson, A. (1966): "Structural Pattern of the Organ of Corti: A Systematic Mapping of Sensory Cells and Neural Elements." Stockholm: Almqvist and Wiksell.

Flock A., Kimura, R., Lundquist, P.-G., and Wersall, J. (1962): Morphological basis of directional sensitivity of the outer hair cells in the organ of Corti. J. Acoust. Soc. Am. 34:135!-1355.

Forge, A. (1985): Outer hair cell loss and supporting cell expansion 
following chronic gentamicin treatment. Hearing Res. 19:171182.

Gabbiani, G., Gabbiani, F.H., and Schwartz, S.M. (1984): Organization of actin cytoskeleton during endothelial regeneration in vitro. J. Cell Sci. 66:39-50.

Geiger, B., Avnur, Z., Rinnerthaler, G., Hinssen, H., and Small, V. (1984): Microfilament organizing centers in areas of cell contact: Cytoskeletal interactions during cell attachment and locomotion. J. Cell Biol. 99:83s-91s.

Geiger, B., Volk, T., and Volberg, T. (1985): Molecular heterogeneity of adherens junctions. J. Cell Biol. 101:1523-1531.

Gulley, R.L., and Reese, T.S. (1976): Intercellular junctions in the reticular lamina or the organ of Corti. J. Neurocytol. 5:479507.

Gumbiner, B. (1987): The structure, biochemistry and assembly of epithelial tight junctions. Am. J. Physiol., 253:C749-C758.

Hawkins, J.E. (1973): Comparative Otopathology: Aging, Noise, and Ototoxic Drugs. Adv. Otorhinolaryngol. 20:125-141.

Hergott, G.J., Sandig, M., and Kalnins, V.I. (1989): Cytoskeletal organization of migrating retinal pigment epithelial cells during wound healing in organ culture. Cell Motil. Cytoskeleton 13: 83-93.

Hsu, S.M., Raine, L., and Fanger, H. (1981): The use of antiavidin antibody and avidin-biotin-peroxidase complex in immunoperoxidase techniques. Am. J. Clin. Pathol. 75:816-821.
Low, R.B., Chaponnier, C., and Gabbiani, G. (1981): Organization of actin in epithelial cells during regenerative and neoplastic conditions. Correlation of morphologic, immunofluorescent and biochemical findings. Lab. Invest. 44:350-367.

McDowell, B., Davies, S., and Forge, A. (1989): The effect of gentamicin induced hair cell loss on the tight junctions of the reticular lamina. Hearing Res. 40:221-232.

Raphael, Y., and Altschuler, R.A. (1990): Scar formation after drug induced cochlear insult. Hearing Res. (in press).

Raphael, Y., Marshak, G., Barash, A., and Geiger, B. (1987): Modulation of intermediate filament expression in the developing cochlear epithelium. Differentiation 35:151-162.

Simons, K., and Fuller, S.D. (1985): Cell surface polarity in epithelia. Annu. Rev. Cell Biol. 1:243-288.

Smith, C.A. (1978): Structure of the cochlear duct. In Naunton, R.F., and Fernandez, C. (eds.): "Evoked Electrical Activity in the Auditory Nervous System." New-York: Academic Press, pp. 3-19.

Takeuchi, S. (1987): The rearrangement of cytoskeletal systems in epithelial cells accompanying the transition from a stationary to a motile state at the start of epithelial spreading. J. Cell Sci. 88:109-119.

Zand, M.S., and Albrecht-Buehler, G. (1989): What structures, besides adhesions, prevent spread cells from rounding up? Cell Motil. Cytoskeleton 13:195-211 